\title{
SYSTEMATIC REVIEW ON GASTROINTESTINAL HELMINTHS OF DOMESTIC RUMINANTS IN ETHIOPIA
}

\author{
Tewodros FENTAHUN \\ Unit of Biomedical Sciences, College of Veterinary Medicine and Animal Sciences, University of Gondar, P.O.Box 196, Gondar, Ethiopia \\ Email: tedyvet@gmail.com; (D) ORCiD: 0000-0002-2955-5638 \\ Supporting Information
}

\begin{abstract}
This systemic review was conducted to identify, critically assess, and bring together available data from primary researches conducted so far on gastrointestinal (GI) helminthes of domestic ruminants in Ethiopia. In the country, GI helminths of domestic ruminants have been identified; examined and informative statistics has been extracted since a few decades ago. For this review, relevant articles were retrieved from English databases: PubMed, Google Scholar, Science Direct, Web of Science and Scientific Information Database (SID). Additional studies were recognized by scanning the African Journal Online (AJOL) that includes the Ethiopian Veterinary Journal and Bulletin of Animal Health and Production. Out of retrieved $(n=154)$ articles, thirty three $(n=37)$ articles which fulfilled the eligibility criteria were selected. Accordingly, twenty three Gl helminthes species which belong to the three classes of helminthes have been found to occur in domestic ruminants in the country. The main genera reported so far are Haemonchus, Strongyloides, Trichostrongylus, Oesophagostomum, Bunostomum, Fasciola, Monezia and Paramphistomum whereas, Haemochus contortus, Moneizia expansa and Fasciolahepatica are the most frequently reported species from Nematode, Cestode and Trematode classes respectively. The overall GI helminths prevalence ranged from $2.3 \%$ to $100 \%$ were reported. Simple flotation, sedimentation, modified McMaster technique and faecal culture are the most common and routine diagnostic methods which have been used in the country. Management aspects like husbandry practices, climate and host influences are found to be the principal contributing factors that affect GI helminths infections. So far, the control of GI parasites in the country is mainly focusing on the use of anthelmintics. Consequently, due to the lack of effective control strategies, antihelmintics are exclusively used which result in antihelmintics resistance. Generally, occurrence, epidemiological features, realistic control strategies, common diagnostic procedures and frequently encountered species are reviewed. Finally, the relevance of epidemiological knowledge and the development of efficient, sustainable and conventional control measures which cover wider ago-climatic zones of the country are suggested for controlling GI helminths infections and should be assessed timely.
\end{abstract}

Keywords: Anthelmintics, Domestic ruminant, Ethiopia, Gastrointestinal helminthes.

\section{INTRODUCTION}

As a result of having different agro-ecological zones and favorable environmental situations in Ethiopia, the country is believed to be endowed numerous livestock species and suitable for livestock production. It has the largest livestock population in Africa (Tilahun and Schmidt, 2012; CSA, 2013). According to CSA (2013) report, an estimated statistics

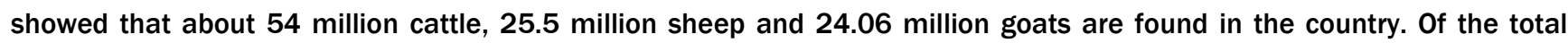
cattle population, $98.95 \%$ are local breeds and the remaining are hybrid and exotic breeds. Furthermore, $99.8 \%$ of the sheep and nearly all goat population of the country are local breeds (CSA, 2013).

However, diseases have numerous negative impacts on production and productivity. Among diverse animal diseases encountered in the country, helminthes infections remain one of the most important limiting factors and a bottlenecking production and productivity these days (Elsa et al., 2012). By chance, the gastrointestinal tract of ruminants harbors variety of parasites particularly helminthes which can cause both clinical and subclinical parasitism. As stated by Lebbie et al. (1994), GI helminthes infections are of a global concern for livestock industry, which have devastating impact in Sub-Saharan Africa in general and in Ethiopia in particular as a result of wider range of agro-ecological factors which are fitting for diversified hosts and parasite species. Hence, gastrointestinal (GI) helminthiasis has become among the most important diseases encountered by livestock sector of Ethiopia and has been considered to be one of the major constraints in the development of the sector (Regassa et al., 2006). In Ethiopia, helminthiasis is responsible for 25\% mortality and 3.8\% weight loss in highland sheep (Bekele et al., 1992). 
According to Zahid et al. (2005), the helminthes infections of ruminants are mostly caused by nematodes like Strongyliodes spp., Ostertagia Spp., Bunostomum and Trichuris spp.; Cestodes such as Moniezia spp., Taenia spp. and Trematodes such as Paramphistomum spp., Fasciola spp. and Shistosoma spp. Perry et al. (2002) reported that GI nematodes have been ranked highest on globalindex with Haemonchus contortus on top. Moreover, Trichostrongyloidea that include genera such as Haemonchus, Trichostrongylus, Cooperia, and Nematodirus, and the Strongyloidea and Ancylostomatoidea with Oesophagostomum and Bunostomum, are the economically most important and widely prevalent GI nematodes (Takele et al., 2013; Winter et al., 2018). In addition, such a pervasive occurrence of the metacestodes; Cysticercus ovis and Cysticercus tenuicollis; Hydatid cyst (E.granulosus); Fasciola hepatic and Fasciola gigantic are most prevalent in the country and considered to be of great economic importance (Lemma et al., 1985; Regassa et al., 2009; Nigatu et al., 2009; Kebede et al., 2009; Feyesa et al., 2010; Nigatu, 2010; Endale et al., 2013; Abebe et al., 2015; Beyene and Hiko, 2019).

There are many associated risk factors influencing the occurrence and epidemiology of GI helminthes including age, sex, weather condition and husbandry or management practices (Khan et al., 2009). Factors such as host age, physiological status, breed, parasite species involved, and the epidemiological patterns (husbandry practices and climate variables) determine the degree of infection (Tembely and Hansen, 1996; Menkir et al., 2007). In Ethiopia, several studies have been conducted on ruminant helminthiasis in various regions reporting a prevalence ranged from 2.3-100\% (Fikru et al., 2006; Abebe and Esayas, 2001; Yirsaw and Zewdu, 2015).

A number of published research reports have been found on GI helminthes of domestic ruminants in Ethiopia. However, these reports are found in a separate and unorganized way. So, comprehensive and well organized documentation about GI helminthes of domestic ruminant in the country is essential to support researchers, professionals and policy-makers to develop further actions on the control and prevention strategies. Therefore, the aim of this systematic review was to identify, assess critically, and bring together available data from primary research conducted so far on gastrointestinal (GI) helminthes of domestic ruminants in Ethiopia.

\section{MATERIALS and METHODS}

\section{Source, selection strategies and protocol}

This systematic review was carried out on GI helminthes of Ethiopian ruminant using available electronic and nonelectronic databases. The electronic search was used as the primary search method. The main electronic databases used were PubMed, Google Scholar, Science Direct, Web of Science, Scientific Information Database (SID) were accessed from University of Gondar, Ethiopia. Relevant studies have been identified from English databases in Pub Med, Google Scholar, Science Direct, Web of Science, Scientific Information Database (SID). Additional studies were recognized by scanning the African Journal Online (AJOL) that includes the Ethiopian Veterinary Journal and Bulletin of Animal Health and Production. Moreover, complete and congress articles like original descriptive studies (designated as cross-sectional study) in sheep, goats and cattle were also considered. Epidemiological parameters such as prevalence of GI helminthes infection among sheep, goat and cattle, and main contributing factors like age, sex, and geographical sub-regions of Ethiopia were considered thoroughly. An intensive data searching was made to collect available information (Figure 3). The searching strategies used were combining the phrases close to GI helminths in large and small ruminants in Ethiopia as indicated in Figure 1. Searches were restricted to peer-reviewed articles published nationally or internationally in English language.

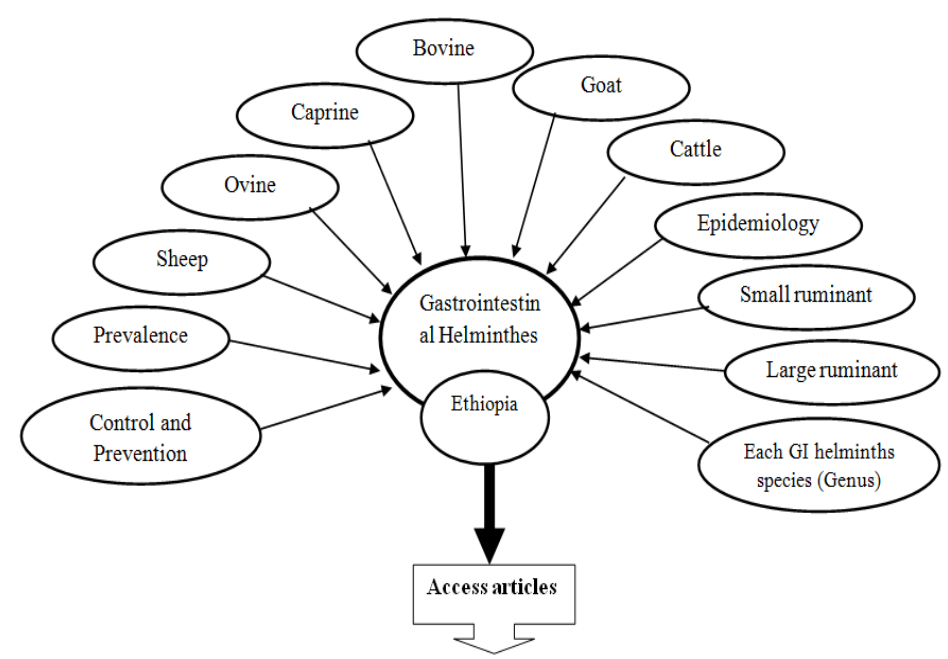




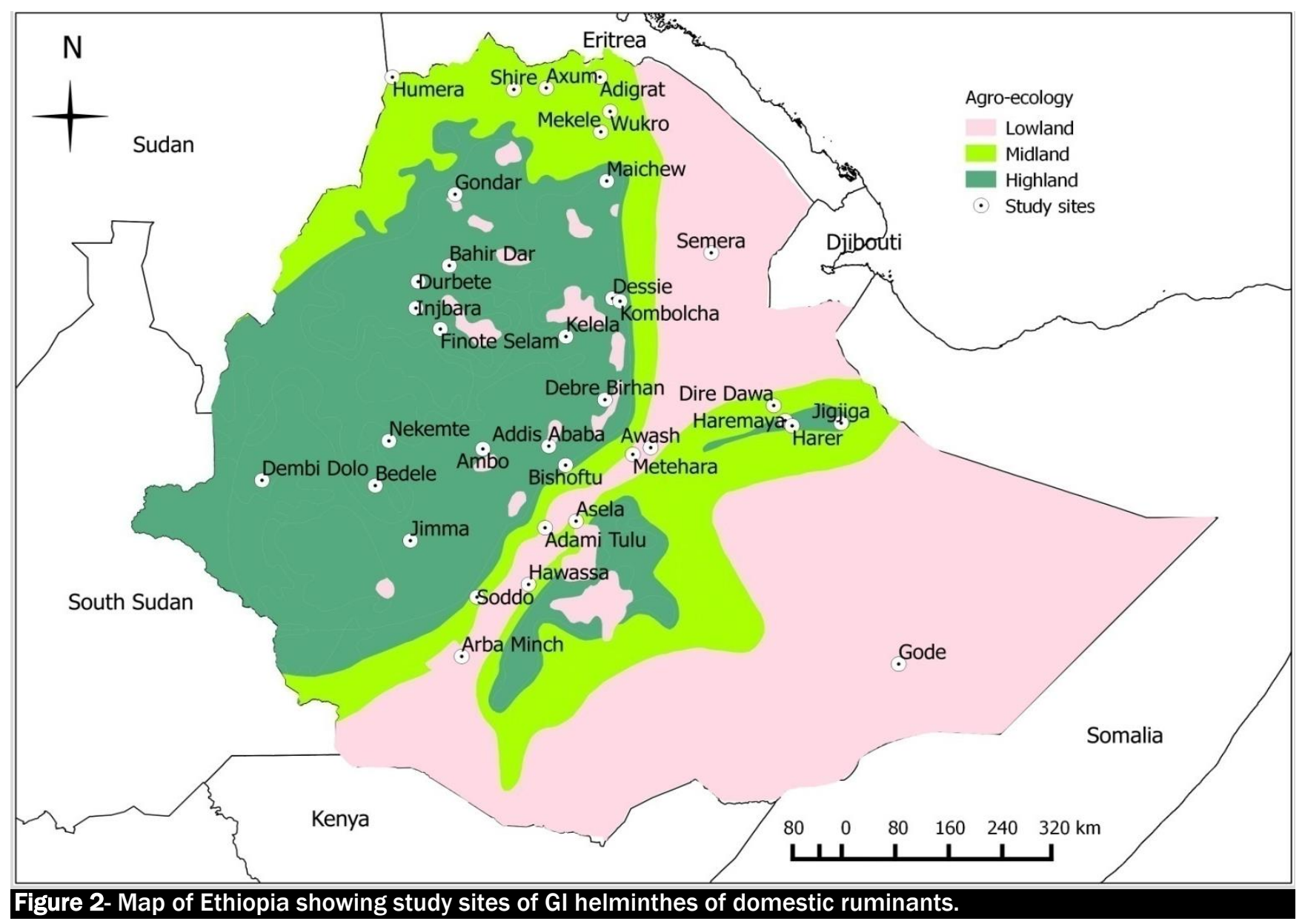

A total of 154 Papers for sheep, goat and cattle from 6 databases (PubMed, Google Scholar, Science Direct, Web of Science, SID, African Journal Online (AJOL) which includes Ethiopian Veterinary Journal and Bulletin of Animal Health and Production .
120 studies which were not dealing with prevalence of GI helminths in domestic ruminants and duplicate papers were excluded.
34 study were selected based on the key words on the topic
Proceedings of Ethiopian Veterinary association (EVA) and annual student and staff conferences of the University of Gondar, Ethiopia and thesis were searched manually.

Figure 3 - Flow diagram describing the study design process and literature search on GI helminthes in domestic ruminants.

\section{Data type and collecting methods}

Published papers were scanned by quick reading to select relevant articles. Important articles were defined and included in the current review as one that contained information on GI helminthes of cattle, sheep and goat in Ethiopia. References of all relevant articles were searched to identify articles that were missed by the electronic search. Any 
identified article was subjected to the same inclusion process as a data type. Following that all relevant articles were reviewed, extracted and compiled in a searchable database (Microsoft Access software, ver. 2007). Extracted information included authors name, study sites, year of the study commenced and ended, year of publication, study design, species of animals, sampling procedure, number of animals selected, body condition, sex, altitude of the study site, laboratory procedure to detect the parasite, testing methodology and prevalence of GI helminthes reported including their associated risk factors.

\section{Reports collected on GI helminths in Ethiopia}

The initial electronic searches yielded a total of $(n=154)$ studies. After scrutinizing these and eliminating duplicates, thirty three $(n=37)$ were considered (Figure 4$)$ including thesis containing relevant information concerning GI helminths of ruminants in Ethiopia. This review work documented that of the relevant articles about $8 \%$ (3) were not retrieved by any of the search engines, $19 \%$ (7) were retrieved by a single search engine, $27 \%(10)$ by two, $16 \%(6)$ by three and $30 \%(11)$ by all four databases. Providing a balanced and an impartial summary of the topic using these representative studies was at the core of this review. No restrictions other than identifying ovine, caprine and bovine GI helminthes in Ethiopia were imposed on the inclusion criteria. This minimized literature selection bias and provided an exhaustive list of GI helminths. However, for factors associated with domestic ruminant GI helminthes in Ethiopia, strict inclusion criteria were applied in order to only select the critical studies. In order for the current review to be critically appraised, repeatability was crucial. This was achieved by documenting every step of the review process.

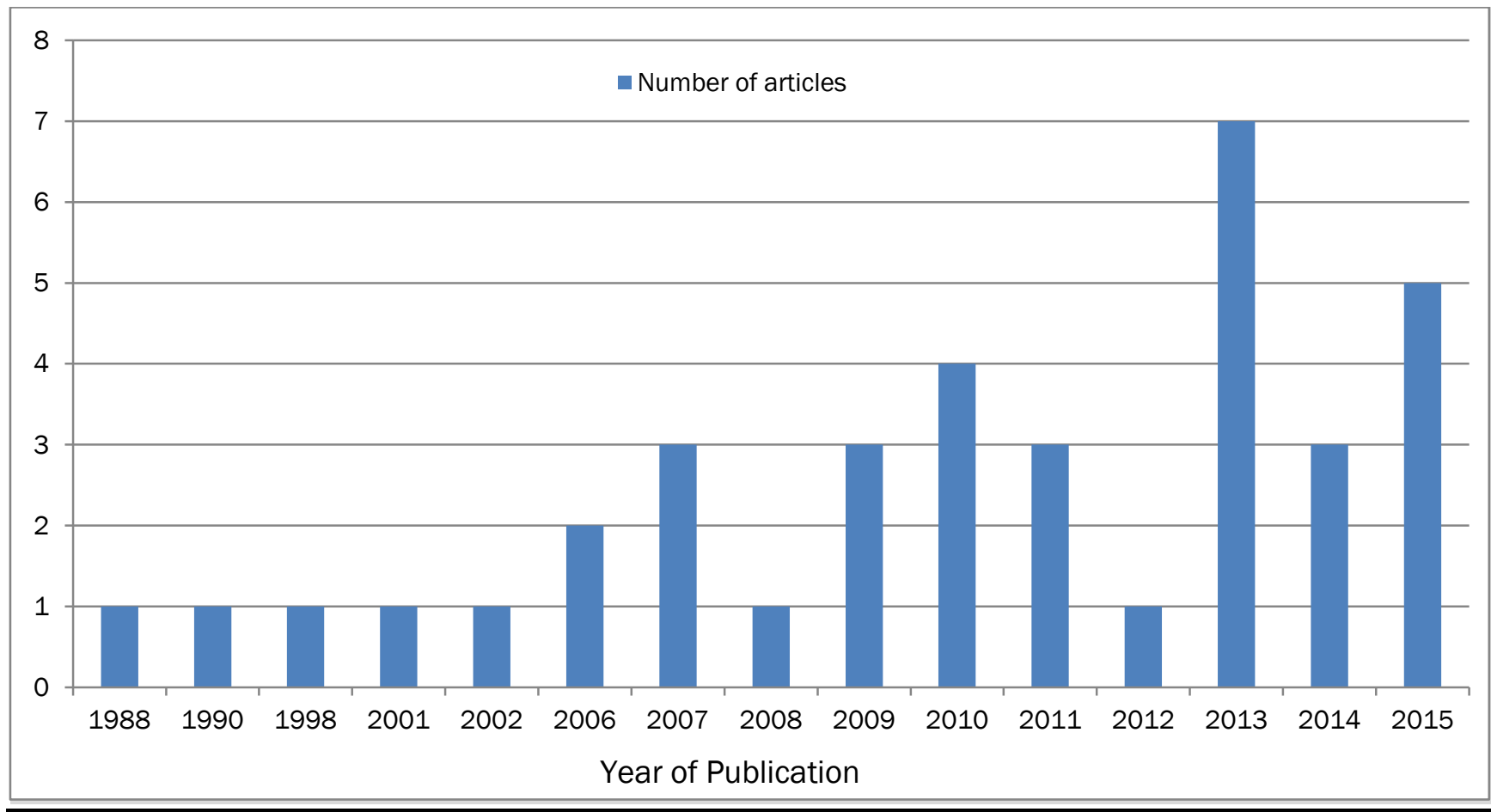

Figure 4 - Frequency of articles relevant to the systematic review on GI helminths of domestic ruminants in Ethiopia.

Occurrence and spatial distribution

The present review work revealed that the occurrences of GI helminthes infections were reported in different parts of the country (Figure 2). The prevalence of GI helminthes is summarized in table 1. The majority of the prevalence studies were specific on some helminthes genera like Strongyles, Fasciola, Shistosoma and some families like GI nematodes, Trematodes and Cestodes. However, adequate sources were not accessed compared to the number of studies done on specific species and families of the GI helminthes of domestic ruminants. In addition, most studies are based on the coprological examinations and few studies were conducted on abattoir surveys in the country.

According to the result obtained from the review, the overall prevalence of GI helminthes is ranged from $13.2 \%$ in Awash River Basin (Afar region) to 81.5\% in Debre Zeit/Bishoftu (Bersissa et al., 2011; Moti et al., 2013). Furthermore, the review underlined that the majority of the identified species are found from nematodes and the lowest from Cestode. On the other hand, Haemochuscontortus, Moneiziaexpansa and Fasciolahepatica are the predominant species from Nematode, Cestode and Trematode classes respectively. Furthermore, Nematodirus spp., Toxocaravitulorum and Schistosoma spp. are less prevalent (Table 2). In Ethiopia the majority of the studies were focusing only on small ruminants' GI helminthes than large ruminants. Therefore, the highest prevalence of the parasite was reported from sheep (91.2\%) in Gode (Ogaden), Southern Ethiopia than cattle (21.9\%) in Nekemete in Western part of Ethiopia (Table 1). On the other hand, most Abattoir survey reports have also shown a status of GI helminths of cattle than small ruminants. 
Table 1 - List of selected studies on GI helminthes of domestic ruminants in Ethiopia

\begin{tabular}{|c|c|c|c|c|c|c|c|c|c|c|c|c|c|}
\hline \multirow{2}{*}{ Study site(s) } & \multicolumn{4}{|c|}{ Study animals and Sample size } & \multirow{2}{*}{ Study period } & \multirow{2}{*}{ ST } & \multirow{2}{*}{$\begin{array}{l}\text { Diagnostic } \\
\text { technique }\end{array}$} & \multicolumn{4}{|c|}{ Prevalence (\%) } & \multirow{2}{*}{ Sample } & \multirow{2}{*}{ Author(year) } \\
\hline & Sheep & Goat & Cattle & Total & & & & Sheep & Goat & Cattle & Total & & \\
\hline Adama & 92 & 208 & 852 & 1152 & Nov2007-Apr2008 & CS & PME & 29.3 & 6.7 & 46.8 & & Liver & Getawa et al. (2010) \\
\hline Adami Tulu & - & 499 & - & 499 & Jul1997-Jun1998 & CS & MM & - & 45.5 & - & 45.5 & $\mathrm{~F}$ & Etana (2002) \\
\hline Addis Ababa & 560 & - & & 560 & Sep 1985 - Jun 1986 & CS & PME & 19.6 & - & - & 19.6 & Liver & Bekele et al.(1988) \\
\hline Ambo & 90 & 30 & - & 120 & Feb-May 2013 & CS & $\mathrm{DS}, \mathrm{FL}, \mathrm{SD}$ & 47.8 & 53.3 & - & - & $\mathrm{F}$ & $\begin{array}{l}\text { Temesgen and Walanso } \\
(2015)\end{array}$ \\
\hline Arba Minch & $241+20$ & $357+25$ & - & $598+45$ & Jan2010-Aug 2011 & CS & $\begin{array}{l}\text { SD,FL, } \\
\text { MM,PME }\end{array}$ & $79.7 ; 85$ & $\begin{array}{c}63.6 ; \\
68\end{array}$ & - & 70.1; & $\mathrm{F}, \mathrm{GIT}$ & Nejib et al. (2014) \\
\hline Asella & - & - & 384 & 384 & Nov 2013-Apr 2014 & CS & $\mathrm{FL}$ & - & - & 49 & 49 & $\mathrm{~F}$ & Addisu and Berihu (2014) \\
\hline Asella & 408 & - & - & 408 & Nov 2008-Apr 2009 & CS & MMM & 68.1 & - & - & 68.1 & $\mathrm{~F}$ & Diriba et al. (2013) \\
\hline Awash & 3,697 & - & - & 3,697 & Jan-Dec 2005 & CS & EAC & 13.2 & - & - & 13.2 & $\mathrm{~F}$ & Ahmed et al.(2007) \\
\hline Bahir Dar & 340 & - & 420 & & $\begin{array}{l}\text { May } 2005 \\
\text { - Dec2006 }\end{array}$ & & & 10.6 & - & 34.05 & - & Liver & Nigatu et al. (2009) \\
\hline Bahir Dar & 384 & - & 384 & 768 & Nov2010-March 2011 & CS & SD, PME & $\begin{array}{l}10.9 ; \\
16.9\end{array}$ & - & $\begin{array}{l}31.5 \\
45.3\end{array}$ & - & F, Liver & $\begin{array}{c}\text { Ayalew and Endalkachew } \\
(2013)\end{array}$ \\
\hline Bahir Dar & - & - & 384 & 4134 & Nov2008-March2009 & CS & SD, PME & & - & 39.95 & 39.95 & F, Liver & Fikirtemariam et al. (2013) \\
\hline Bahir Dar & - & - & 384 & 384 & Nov2013-April 2014 & CS & SD & 32.3 & - & - & 32.3 & $\mathrm{~F}$ & Yitayal et al. (2015) \\
\hline Bedele & - & - & 500 & 500 & Oct 2011-March 2012 & CS & $\mathrm{SD}, \mathrm{FL}, \mathrm{MM}$ & - & - & 64.2 & 64.2 & $\mathrm{~F}$ & Moti et al. (2013) \\
\hline Bishoftu (Debre Zeit) & 1152 & 1536 & - & 2688 & Dec2005 - Jun 2006 & CS & PME & 58.5 & 43.8 & - & & Liver & Jibat et al. (2008) \\
\hline Bishoftu (Debre Zeit) & 157 & 65 & - & 222 & Nov2007-Apr2008 & CS & $\mathrm{FL}, \mathrm{SD}, \mathrm{CC}$ & 81.0 & 83.0 & - & 81.5 & $\mathrm{~F}$ & Bersissa et al. (2011) \\
\hline Bishoftu (Debre Zeit) & - & - & 326 & 326 & Nov2011-Apr 2012 & CS & $\mathrm{FL}, \mathrm{SD}$, & - & - & 61 & 61 & $\mathrm{~F}$ & Cheru et al. (2014) \\
\hline Debre Berhan & 2500 & - & - & 2500 & 1987 & CS & $\mathrm{SD}, \mathrm{FL}$ & 18 & - & - & 18 & F, PME & Njau et al.(1990) \\
\hline Dembi Dolo & 255 & 245 & 257 & 757 & 2003-2004 & CS & $\mathrm{DS}, \mathrm{SD}, \mathrm{FL}, \mathrm{MM}$ & 75.3 & 84.1 & 50.2 & 69.6 & $\mathrm{~F}$ & Fikru et al.(2006) \\
\hline Dessie & 510 & 420 & - & 930 & Nov2011-Mar 2012 & CS & PME & 21.04 & 27.61 & - & - & OM, PE, Liver,MS & Abebe et al.(2015) \\
\hline Dire Dawa & 425 & 420 & - & 845 & Nov2011-April 2012 & CS & PME & 22.8 & 26.4 & - & 24.6 & Liver, PE OM & Endale et al. (2013) \\
\hline Durbete & $202-$ & - & 330 & 532 & Oct 2014- Apr 2015 & CS & $\mathrm{SD}$ & 2.3 & - & 24.6 & 26.9 & $\mathrm{~F}$ & Yirsaw and Zewdu (2015) \\
\hline Gondar & 458 & 100 & & 558 & Nov-Jan 2008 & CS & $\mathrm{SD}, \mathrm{FL}$ & 46.07 & 55 & 2.0 & 47.67 & $\mathrm{~F}$ & Shimelis et al. (2011) \\
\hline Gondar, Finote Selam, Injibara & - & - & 22,755 & 22,755 & Sept 2002-2007 & CS & PME & - & - & 79.5 & 79.5 & Liver & Nigatu (2010) \\
\hline $\begin{array}{l}\text { Haremaya, Harar, Dire Dawa, } \\
\text { Jijiga }\end{array}$ & 655 & 632 & - & 1,287 & May 2003-Apr 2005 & CS & PME & & & & 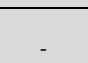 & GIT & Menkir et al. (2007) \\
\hline Hawassa & 284 & 226 & - & 510 & Nov2008-Feb 2009 & CS & MMM & 47.2 & 38.9 & - & - & $\mathrm{F}$ & Abebe et al. (2010) \\
\hline Hawassa & - & - & 632 & 632 & Dec2008 - Mar 2009 & CS & PME & - & - & 52.6 & 52.6 & Liver & Feyesa et al. (2010) \\
\hline Hawassa & 180 & 132 & - & 312 & Jan-Jun 2006 & CS & PME & 91.1 & 87.1 & - & & $A$ & Thomas et al. (2007) \\
\hline Jimma & - & - & 210 & 210 & Nov2008-Apr 2009 & CS & $\mathrm{FL}, \mathrm{SD}, \mathrm{MMM}$ & - & - & 77.6 & 77.6 & $\mathrm{~F}$ & Hailu et al. (2011) \\
\hline Kelala & 230 & - & 154 & 384 & Sept1997-Apr1998 & CS & $\mathrm{FL}, \mathrm{SD}$ & 53 & - & 38 & - & $\mathrm{F}$ & Tesfaye (1998) \\
\hline Kombolcha & - & - & 400 & 400 & 2011 & CS & PME & - & - & 17 & 17 & Liver & Fufa et al. (2012) \\
\hline Mekelle & - & - & 1023 & 1023 & Nov2007 - Feb2008 & CS & PME & - & - & 7.23 & 7.23 & Liver & $\begin{array}{l}\text { Getachew and Ashwani } \\
\text { (2013) }\end{array}$ \\
\hline $\begin{array}{l}\text { Mekelle, Adigrat, Axum, } \\
\text { Humera, Maichew, Shire }\end{array}$ & - & - & 5,194 & 5,194 & $2007 / 8$ & CS & PME & - & - & 22.1 & 22.1 & Liver & Kebede et al. (2009) \\
\hline Metehara, Semera, Jigjiga & 92 & 91 & - & 183 & Nov1998-Apr 1999 & CS & MMM, PME & 97.03 & 100 & - & & $\mathrm{F}, \mathrm{GIT}$ & Abebe and Esayas(2001) \\
\hline Nekemete & - & - & 384 & 384 & Nov2011-March 2012 & CS & PME & - & - & 21.9 & 21.9 & GIT & Alula et al. (2013) \\
\hline Ogaden-Gode & 114 & 82 & - & 196 & Aug2003-March 2004 & $\mathrm{CS}$ & PME & 91.2 & 82.9 & - & - & A & Kumsa and Wossene (2006) \\
\hline Wolaita Soddo & - & - & 415 & 415 & Nov2007 - Apr2008 & CS & PME & - & - & 11.3 & - & Liver & Regassa et al. (2009) \\
\hline Wukro & 384 & - & - & 384 & Nov 2013-Apr 2014 & CS & PME & 40.9 & - & - & 40.9 & A & Lidya and Berihun (2015) \\
\hline
\end{tabular}


Table 2 - GI helminthes species/genus composition; collected from each selected studies in Ethiopia.

\begin{tabular}{|c|c|c|c|c|c|c|c|c|c|c|c|c|c|c|}
\hline \multicolumn{12}{|c|}{ Species of parasite identified (\%) } & \multirow{3}{*}{ Location } & \multirow{3}{*}{$\begin{array}{c}\text { Altitude } \\
\text { of study } \\
\text { area }\end{array}$} & \multirow{3}{*}{$\begin{array}{l}\text { Author } \\
\text { (year) }\end{array}$} \\
\hline \multirow{2}{*}{ Nematode } & \multicolumn{3}{|c|}{ Species of animal } & \multirow{2}{*}{ Cestode } & \multicolumn{3}{|c|}{ Species of animal } & \multirow{2}{*}{ Trematode } & \multicolumn{3}{|c|}{ Species of animal } & & & \\
\hline & Sheep & Goat & Cattle & & Sheep & Goat & Cattle & & Sheep & Goat & Cattle & & & \\
\hline - & - & - & 852 & Hydatid cyst & 40.0 & 55.6 & 37.1 & - & - & - & - & Adama & 1770 & $\begin{array}{l}\text { Getaw et al. } \\
(2010)\end{array}$ \\
\hline H.contortus & - & 30.3 & - & \multirow{7}{*}{$\begin{array}{l}\text { M. expansa } \\
\text { M. benedeni }\end{array}$} & & & & & & & & \multirow{7}{*}{ Adami Tulu } & \multirow{7}{*}{900} & \multirow{7}{*}{ Etana (2002) } \\
\hline Strongyloidesspp. & - & 16.4 & - & & & & & & & & & & & \\
\hline Trichostrongylus spp. & - & 13.8 & - & & - & 14.6 & - & - & - & - & - & & & \\
\hline Trichuris spp. & - & 2.2 & - & & & & & & & & & & & \\
\hline Nematodirus spp & - & 2.8 & - & & - & 4.0 & - & - & - & - & - & & & \\
\hline Chabertia ovina & - & 5.0 & - & & & & & & & & & & & \\
\hline Bunostomum spp. & - & 5.4 & - & & & & & & & & & & & \\
\hline 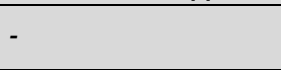 & - & - & - & $\begin{array}{l}\text { T.hydatigena and } \\
\text { Hydatid cyst }\end{array}$ & 18.6 & - & - & - & - & - & & $\begin{array}{l}\text { Addis } \\
\text { Ababa }\end{array}$ & 2,326 & $\begin{array}{l}\text { Bekele et al. } \\
\text { (1988) }\end{array}$ \\
\hline Strongyles spp. & 47.8 & 53.3 & - & - & - & - & - & Fasciola spp. & 4.4 & 6.7 & - & Ambo & 2185 & $\begin{array}{l}\text { Temesgen and } \\
\text { Walanso (2015) }\end{array}$ \\
\hline $\begin{array}{l}\text { H.contortus } \\
\text { Trichostrongylus spo. }\end{array}$ & $\begin{array}{l}A^{*} \\
A^{*}\end{array}$ & $\begin{array}{l}A^{*} \\
A^{*}\end{array}$ & - & - & & & & & & & & \multirow{4}{*}{ Arba Minch } & \multirow{4}{*}{1400} & \multirow{4}{*}{ Nejib et al. (2016) } \\
\hline Teladorsagia spp. & $A^{*}$ & $A^{*}$ & - & - & & & & & & & & & & \\
\hline T.colubriformis & $A^{*}$ & $A^{*}$ & - & - & & & & & & & & & & \\
\hline Haemonchus spp. & $A^{*}$ & $A^{*}$ & - & - & & & & & & & & & & \\
\hline Ostertagiaspp & - & - & 1.8 & & & & & & & & & \multirow{6}{*}{ Assela } & \multirow{6}{*}{$\begin{array}{l}1500- \\
2300\end{array}$} & \multirow{6}{*}{$\begin{array}{l}\text { Addisu and Berihu } \\
\qquad(2014)\end{array}$} \\
\hline Oesophagostomumsp & - & - & 1.3 & & & & & & & & & & & \\
\hline Strongloid spp. & - & - & 2.6 & & & & & & & & & & & \\
\hline Trychostrongylusspp & - & - & 3.6 & & & & & & & & & & & \\
\hline Hemonchusspp. & - & - & 11.7 & & & & & & & & & & & \\
\hline Bunostomum spp. & - & - & 4.4 & & & & & & & & & & & \\
\hline Strongylesspp & 64.0 & - & - & & & & & & & & & \multirow{3}{*}{ Asella } & & \multirow{3}{*}{$\begin{array}{l}\text { Diriba et al. } \\
\text { (2013) }\end{array}$} \\
\hline Strongyloidesspp & 7.4 & - & - & & & & & & & & & & 3000 & \\
\hline Trichurisspp & 3.7 & - & - & & & & & & & & & & & \\
\hline- & - & - & - & - & - & - & - & Fasciola spp. & $13.2 \%$ & - & - & Awash & $550-850$ & $\begin{array}{l}\text { Ahmed et al. } \\
\text { (2007) }\end{array}$ \\
\hline- & - & - & - & E. granulosus & 25.0 & - & 16.1 & - & - & - & - & Bahir Dar & 1784 & $\begin{array}{l}\text { Nigatu et al. } \\
(2009)\end{array}$ \\
\hline- & - & - & - & - & - & - & - & $\begin{array}{l}\text { F. hepatica and } \\
\text { F.gigantica }\end{array}$ & 16.9 & - & 45.31 & Bahir Dar & 1784 & $\begin{array}{c}\text { Ayalew and } \\
\text { Endalkachew } \\
(2013)\end{array}$ \\
\hline - & - & - & - & - & - & - & - & $\begin{array}{l}\text { F.hepatica } \\
\text { F.gigantic } \\
\text { Mixed }\end{array}$ & $\begin{array}{l}- \\
- \\
-\end{array}$ & $\begin{array}{l}- \\
- \\
-\end{array}$ & $\begin{array}{l}89.7 \\
3.63 \\
6.67\end{array}$ & Bahir Dar & 1784 & $\begin{array}{l}\text { Fikirtemariam et } \\
\text { al. (2013) }\end{array}$ \\
\hline- & - & - & - & - & - & - & - & F. hepatica & - & - & 32.3 & Bahir Dar & 1784 & $\begin{array}{l}\text { Yitayal et al. } \\
\text { (2015) }\end{array}$ \\
\hline $\begin{array}{l}\text { Trichuris spp. } \\
\text { Trichostrongyles spp. }\end{array}$ & - & - & $\begin{array}{c}6.8 \\
11.0\end{array}$ & - & $\overline{-}$ & - & $\overline{-}$ & $\begin{array}{c}\text { Fasciola spp } \\
\text { Paramphistomu spp, }\end{array}$ & $\begin{array}{l}- \\
-\end{array}$ & $\begin{array}{l}- \\
-\end{array}$ & $\begin{array}{l}51.4 \\
18.7\end{array}$ & Bedele & 2060 & Moti et al. (2013) \\
\hline 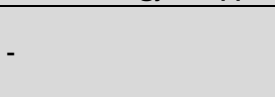 & - & - & - & $\begin{array}{l}\text { St.hepatica } \\
\text { C.tenuicollis }\end{array}$ & $\begin{array}{l}9.5 \\
5.2\end{array}$ & $\begin{array}{l}12.1 \\
8.3\end{array}$ & - & Fasciola spp. & 6.9 & 3.6 & - & Bishoftu & 2155 & Jibat et al. (2008) \\
\hline $\begin{array}{l}\text { Strongyle type } \\
\text { Strongyloides spp. }\end{array}$ & $\begin{array}{c}56.60 \\
8.2\end{array}$ & $\begin{array}{l}61.0 \\
15.4\end{array}$ & $\begin{array}{l}- \\
-\end{array}$ & M.expansa & 13 & 29.2 & - & Fasciolaspp. & 0.63 & 0 & - & Bishoftu & 2155 & $\begin{array}{l}\text { Bersissa et al. } \\
\text { (2011) }\end{array}$ \\
\hline
\end{tabular}




\begin{tabular}{|c|c|c|c|c|c|c|c|c|c|c|c|c|c|c|}
\hline Trichuris spp. & 5.0 & 0.0 & - & & & & & & & & & & & \\
\hline $\begin{array}{l}\text { Strongyle spp. } \\
\text { Trichuris spp. } \\
\text { Toxocara spp. } \\
\text { Trichuris spp. }\end{array}$ & $\begin{array}{l}- \\
- \\
- \\
-\end{array}$ & $\begin{array}{l}- \\
- \\
- \\
-\end{array}$ & $\begin{array}{c}41.4 \\
41.4 \\
5.2 \\
5.2\end{array}$ & Monezia spp. & - & - & 2.8 & $\begin{array}{l}\text { Fasciola spp. } \\
\text { Paramphistomum } \\
\text { spp. }\end{array}$ & $\begin{array}{l}- \\
-\end{array}$ & $\begin{array}{l}- \\
-\end{array}$ & $\begin{array}{l}36.5 \\
18.4\end{array}$ & Bishoftu & 2155 & $\begin{array}{l}\text { Cheru et al. } \\
\text { (2014) }\end{array}$ \\
\hline Trichuris ovis & 25.4 & - & - & & & & & & & & & & & \\
\hline $\begin{array}{l}T \text {, axei } \\
\text { H. contortus }\end{array}$ & $\begin{array}{c}19.4 \\
2.9\end{array}$ & - & - & $\begin{array}{c}\text { C. ovis } \\
\text { Moniezia spp. }\end{array}$ & $\begin{array}{l}11.9 \\
7.5\end{array}$ & - & - & Fasciola hepatica & $4.5 \%$ & - & - & $\begin{array}{l}\text { Debre } \\
\text { Berhan }\end{array}$ & $\begin{array}{c}2500- \\
300\end{array}$ & Njau et al. (1990) \\
\hline Ostertagia spp. & 1.5 & - & - & & & & & & & & & & & \\
\hline Ascaris spp. & 1.6 & 2.5 & 2.8 & & & & & & & & & & & \\
\hline $\begin{array}{l}\text { Strongyle spp. } \\
\text { Dictyocaulus spp. }\end{array}$ & $\begin{array}{c}70.2 \\
4.3\end{array}$ & $\begin{array}{c}78.4 \\
1.2\end{array}$ & $\begin{array}{l}47 \\
0.4\end{array}$ & - & & & & Fasciola spp. & 0 & 0 & 0.4 & $\begin{array}{l}\text { Dembi } \\
\text { Dolo }\end{array}$ & $\begin{array}{l}1500- \\
2000\end{array}$ & Fikru et al. (2006) \\
\hline Trichuris spp. & 4.5 & 9.8 & 1.6 & & & & & & & & & & & \\
\hline - & - & - & - & $\begin{array}{l}\text { C.tenuicollis, C.ovis } \\
\text { Hydatid cysts }\end{array}$ & $\begin{array}{l}45.69 \\
8.43 \\
9.02\end{array}$ & $\begin{array}{c}72.38 \\
8.57 \\
1.90\end{array}$ & $\begin{array}{l}- \\
- \\
-\end{array}$ & - & - & - & - & Dessie & 2,400 & $\begin{array}{l}\text { Abebe et al. } \\
\text { (2015) }\end{array}$ \\
\hline - & - & - & - & C.tenuicollis & 22.8 & 26.4 & - & - & - & - & - & Dire Dawa & 1204 & $\begin{array}{c}\text { Endale et al. } \\
(2013)\end{array}$ \\
\hline Shistisoma spp. & 2.3 & - & 24.6 & - & - & - & - & - & - & - & - & Durbete & 1600 & $\begin{array}{c}\text { Yirsaw and Zewdu } \\
\text { (2015) }\end{array}$ \\
\hline $\begin{array}{l}\text { Strongyle spp. } \\
\text { Trichuris spp. }\end{array}$ & $\begin{array}{c}34.06 \\
4.8\end{array}$ & $\begin{array}{c}54 \\
3\end{array}$ & - & Monezia Spp. & 3.93 & 0 & - & $\begin{array}{c}\text { F. hepatica } \\
\text { Paramphistomum } \\
\text { spp. }\end{array}$ & $\begin{array}{l}8.52 \\
0.87\end{array}$ & $\begin{array}{c}6.99 \\
0\end{array}$ & - & Gondar & $\begin{array}{l}1500- \\
2000\end{array}$ & $\begin{array}{c}\text { Shimelis et al. } \\
\text { (2011) }\end{array}$ \\
\hline - & - & - & - & Hydatid cysts & - & - & 38.8 & - & - & - & - & $\begin{array}{l}\text { Gondar, } \\
\text { Finote } \\
\text { Selam, } \\
\text { Injibara }\end{array}$ & $\begin{array}{l}1917- \\
2560\end{array}$ & Nigatu (2010) \\
\hline H contortus & & $\Delta *$ & 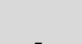 & M. expansa & 61 & 53 & & & & & & & & \\
\hline $\begin{array}{l}\text { H.contortus. } \\
\text { T.axei }\end{array}$ & $\begin{array}{l}A^{*} \\
A^{*}\end{array}$ & $\begin{array}{l}A^{*} \\
A^{*}\end{array}$ & - & & & & & & & & & & & \\
\hline T.vitrinus & $A^{*}$ & $A^{*}$ & - & Av.centripunctata & 20 & 21 & & P.microbothrium & 25 & 21 & & & & \\
\hline Trichostrongylus spp. & $A^{*}$ & $A^{*}$ & - & & & & & & & & & & & \\
\hline Nematodirus spathir & $A^{*}$ & $A^{*}$ & - & St.globipunctata & 24 & 27 & & F.hepatica & & & & Haremaya, & & \\
\hline Cooperia curticei & $A^{*}$ & $A^{*}$ & - & & & & & & 26 & 3 & & Harar, Dire & & Menkir et al. \\
\hline Strongyloides spp. & $A^{*}$ & $A^{*}$ & - & St.hepatica & 39 & 36 & & F. gigantica & & - & & Dawa, & 2000 & $(2007)$ \\
\hline Bunostomum spp & $A^{*}$ & $A^{*}$ & - & Cvsticercus ovis) & 26 & 22 & & & 20 & 10 & & Jijiga & & \\
\hline Oes.columbianum & $A^{*}$ & $A^{*}$ & - & cysticercus ovis) & 26 & 22 & & Dicrocoelium & & & & & & \\
\hline Oesophagostomum & $A^{*}$ & $A^{*}$ & - & Ctenuicollis & 79 & 53 & & dendriticum & 7 & 2 & & & & \\
\hline Chabertia ovina & $A^{*}$ & $A^{*}$ & - & C.tenuicollis & 19 & 53 & & & & & & & & \\
\hline Trichuris spp & $A^{*}$ & $A^{*}$ & - & E. granulosus (cysts) & 68 & 65 & & & & & & & & \\
\hline Haemonchus spp. & 51.6 & 64.7 & & & & & & & & & & & & \\
\hline Trichostrongylus spp. & 38.7 & 41.2 & & & & & & & & & & & & \\
\hline Oesophagostomum & 22.6 & 23.5 & & & & & & & & & & Hawassa & 1790 & Abebe et al. \\
\hline Trichuris spp. & 27.6 & 12.5 & & & & & & & & & & & & \\
\hline Bunostomum spp. & 9.7 & 11.8 & & & & & & & & & & & & \\
\hline - & - & - & - & Hydatid cysts & - & - & 6.9 & 6.9 & - & - & & Hawassa & 1790 & $\begin{array}{l}\text { Feyesa et al. } \\
(2010)\end{array}$ \\
\hline Haemonchus spp. & 81.1 & 76.5 & - & & & & & & & & & & & \\
\hline T.axei & 47.2 & 39.4 & - & & & & & & & & & Hawassa & 1790 & Thomas et al. \\
\hline Teladorsagia spp. & 19.4 & 20.5 & - & & & & & & & & & & & \\
\hline
\end{tabular}




\begin{tabular}{|c|c|c|c|c|c|c|c|c|c|c|c|c|c|c|}
\hline $\begin{array}{l}\text { Strongyels spp. } \\
\text { Strongyloid spp. } \\
\text { Trichuris spp. } \\
\text { Ascaris spp. } \\
\text { Capillaria spp. } \\
\text { Nematodirus spp. }\end{array}$ & $\begin{array}{l}- \\
- \\
- \\
- \\
-\end{array}$ & $\begin{array}{l}- \\
- \\
- \\
- \\
-\end{array}$ & $\begin{array}{c}32.4 \\
3.3 \\
1.9 \\
2.4 \\
1.4 \\
0.9\end{array}$ & M. expansa & - & - & 5.2 & $\begin{array}{c}\text { Fasciola spp. } \\
\text { Paramphistomum }\end{array}$ & - & - & $\begin{array}{l}23.3 \\
48.6\end{array}$ & Jimma & 1750 & Hailu et al. (2011) \\
\hline $\begin{array}{l}\text { Strongyles spp. } \\
\text { Trichuris spp. } \\
\text { Strongyloides spp. } \\
\text { Nematodirus spp. }\end{array}$ & $\begin{array}{l}27.58 \\
2.95 \\
3.20 \\
0.49\end{array}$ & $\begin{array}{l}- \\
- \\
-\end{array}$ & $\begin{array}{c}3.6 \\
0.74 \\
0.6 \\
5.3\end{array}$ & Moniezia sp. & 4.18 & - & 0.4 & F. hepatica & 1.47 & - & 4.8 & Kelala, & 1580 & Tesfaye (1998) \\
\hline - & - & - & - & Hydatid cysts & - & - & 4.25 & - & - & - & - & Kombolcha & $\begin{array}{l}500- \\
1840\end{array}$ & Fufa et al. (2012) \\
\hline - & - & - & - & Cysticercus bovis & - & - & 6. 74 & - & - & - & - & Mekelle & $\begin{array}{l}2000- \\
2200\end{array}$ & $\begin{array}{l}\text { Getachew and } \\
\text { Ashwani (2013) }\end{array}$ \\
\hline - & - & - & - & $\begin{array}{l}\text { Hydatid cyst } \\
\text { (E.granulosus) }\end{array}$ & - & - & 8.1 & - & - & - & - & $\begin{array}{c}\text { Mekelle, } \\
\text { Adigrat, Axum } \\
\text { Humera, } \\
\text { Maiche, Shire }\end{array}$ & $\begin{array}{l}1400- \\
2500\end{array}$ & $\begin{array}{l}\text { Kebede et al. } \\
\text { (2009) }\end{array}$ \\
\hline $\begin{array}{l}\text { Haemonchus spp. } \\
\text { T.axei } \\
\text { T.colubriformis } \\
\text { Bunostomum spp. } \\
\text { Strongyloides spp. } \\
\text { Oesophagostomm. } \\
\text { Trichuris spp. }\end{array}$ & \begin{tabular}{c|}
90.82 \\
55.8 \\
87.15 \\
38.97 \\
38.02 \\
74.8 \\
51.75
\end{tabular} & \begin{tabular}{|c|}
96.5 \\
64.3 \\
90 \\
35.2 \\
43.6 \\
70.8 \\
48.18
\end{tabular} & - & $\begin{array}{c}\text { Avitellina spp. } \\
\text { Stilesia spp. }\end{array}$ & $\begin{array}{l}27.6 \\
32.8\end{array}$ & $\begin{array}{l}20.6 \\
34.0 \\
35.1 \\
28.8\end{array}$ & - & - & - & - & - & $\begin{array}{l}\text { Metehara, } \\
\text { Semera, } \\
\text { Jigjiga }\end{array}$ & 2300 & $\begin{array}{l}\text { Abebe and Esayas } \\
\text { (2001) }\end{array}$ \\
\hline - & - & - & - & - & - & - & & Fasciola spp. & - & - & 21.9 & Nekemete & 2,088 & Alula et al.(2013) \\
\hline $\begin{array}{l}\text { Haemonchus spp. } \\
\text { Trichostrongylus spp. }\end{array}$ & $\begin{array}{l}91.2 \\
37.7\end{array}$ & $\begin{array}{l}82.9 \\
40.2\end{array}$ & - & - & - & - & - & - & - & - & - & Ogaden & 1200 & $\begin{array}{c}\text { Kumsa and } \\
\text { Wossene (2006) }\end{array}$ \\
\hline - & - & - & - & $\begin{array}{c}\text { C. bovis } \\
\text { Hydatid cyst }\end{array}$ & $\begin{array}{l}- \\
-\end{array}$ & - & $\begin{array}{l}0.0 \\
9.9\end{array}$ & - & - & - & - & $\begin{array}{l}\text { Wolaita } \\
\text { Soddo }\end{array}$ & 2500 & $\begin{array}{l}\text { Regassa et al. } \\
\text { (2009) }\end{array}$ \\
\hline H.contortus & 40.9 & - & - & - & - & - & - & - & - & - & - & Wukro & 1977 & $\begin{array}{l}\text { Lidya and Berihun } \\
\text { (2015) }\end{array}$ \\
\hline
\end{tabular}




\section{Species composition and seasonal dynamics}

In Ethiopia, the presence of GI helminthes infections in domestic ruminants is reported by many authors (Thomas et al., 2007; Menkir et al., 2007; Abebe et al., 2010; Shimelis et al. 2011; Diriba et al., 2013; Addisu and Berihu, 2014 and Cheru et al. 2014). According to the current review, about twenty three (23) GI helminthes species belonging to Nematode, Cestode and Trematode categories have been reported from infected cattle, sheep and goats (Table 2). Whereas, Menkir et al. (2007), Abebe et al. (2010), Hailu et al. (2011) and Shimelis et al. (2011) who reported that the most prevalent genera of GI helminthes were Haemonchus, Trichostronglyus. Oesophagostomum, Nematodirus, Cooperia, Toxocara and Bunostomum from Nematodes; Monezia and Cyticercusfrom Cestodes and Fasciola, Paramphistomum and Shistosoma from Trematode classes (Table 3).

Table 3 - Summary of Species compositions of GI helminthes of domesticated ruminants in Ethiopia.

\begin{tabular}{|c|c|c|c|}
\hline Major Class & Species & Host & Predilection site(s) \\
\hline \multirow{15}{*}{ Nematode } & Haemonchus contortus & Cattle, sheep, goats & Abomasums \\
\hline & Haemonchus placei & Cattle & Abomasums \\
\hline & Ostertagia circumcincta & Cattle, Sheep, Goats & Abomasums \\
\hline & Trichostrongylus axei & Cattle, Sheep, Goats & Small intestine \\
\hline & Trichostrongylus colubriformis & Cattle, Sheep, Goats & Small intestine \\
\hline & Cooperia curticei & Cattle, Sheep, Goats & Small intestine \\
\hline & Strongyloides papillosus & Cattle, Sheep, Goats & Small intestine \\
\hline & Ostertagia Spp. & Cattle, Sheep, Goats & Abomasum, Large intestine \\
\hline & Chabertiaovina & Cattle, Sheep, Goats & Large intestine \\
\hline & Oesophagostomum columbianum & Sheep, Goat & Large intestine \\
\hline & Strongyloidespapillosus & Cattle, Sheep, Goats & Small intestine \\
\hline & Trichuris ovis & Cattle, Sheep, Goats & Large intestine \\
\hline & Nematodirus filicollis & Cattle, Sheep, Goats & Small intestine \\
\hline & Nematodirus spathiger & Sheep & Small intestine \\
\hline & Trichostrongylus vitrinus & $\begin{array}{l}\text { Sheep, Goat } \\
\text { Cattle (calves) }\end{array}$ & $\begin{array}{l}\text { Small intestine } \\
\text { Small intestine }\end{array}$ \\
\hline \multirow[t]{7}{*}{ Cestode } & Monezia expansa & Sheep, Goat & Small intestine \\
\hline & Cysticercustenuicollis (Taenia hydatigena) & Sheep, Goat & Omentum Mesenteries Peritoneum, Liver \\
\hline & Ecchnoccocus granulossus & Sheep, Goat & Omentum Mesenteries Peritoneum, Liver \\
\hline & Cysticercus ovis (Taenia ovis) & Sheep, Goat & Omentum Mesenteries Peritoneum, Liver \\
\hline & Avitellina centripunctata (Av.centripunctata) & Sheep, Goat & Small intestine \\
\hline & Stilesia globipunctata (St.globipunctata) & Sheep, Goat & Small intestine \\
\hline & Stilesia hepatica (St. hepatica) & Sheep, Goat & Liver, biliary ducts \\
\hline \multirow{5}{*}{ Trematode } & Fasciola hepatica & Sheep, Goat Cattle & Liver \\
\hline & Fasciola gigantica & Sheep, Goat Cattle & Liver \\
\hline & Paramphistomum cervi & Sheep, Goat Cattle & Rumen \\
\hline & Paramphistomum microbothrium & Sheep, Goat Cattle & Rumen \\
\hline & Schistosoma bovis & Sheep, Goat Cattle & $\begin{array}{l}\text { Liver, intestine, mesenteric lymph nodes } \\
\text { and mesenteric veins }\end{array}$ \\
\hline
\end{tabular}

According to this systemic review, GI helminthiasis in domestic ruminants is severe and increasingly become an important focusing area of research in the country situation. A study conducted in western Oromia on GI parasites showed that Strangles were the most prevalent parasites encountered in the area (Moti et al., 2013). Similar study by Shimelis et al. (2011); Cheru et al. (2014), Nejib et al. (2016) and Diriba et al. (2013) reported that Strongyles were the most prevalent parasites encountered in North Gondar, Debre Zeit (East Shoa zone), Arbaminch (GamoGofa zone) and Asella respectively. As it has been reported by Abebe et al. (2015); Endale et al. (2013); Feyesa et al. (2010); Nigatu (2010); Kebede et al. (2009); Nigatu et al. (2009); Regassa et al. (2009); Jibat et al. (2008); Menkir et al. (2007); Bekele et al. (1988), metacestodes (larval cestodes) Cysticercus ovis (Taeniaovis), Cysticercus tenuicollis (T. hydatigena) and Hydatid cysts (Echinococcus granulosus) are the most prevalent species in Eastern Ethiopia. On the other hand, gastrointestinal infections as a result of adult cestodes such as Avitellina centripunctata, Moniezia expansa and Stilesia globipunctata, and bile duct infections with Stilesia hepatica were frequently reported in different parts of the country (Abebe and Esayas, 2001; Etana, 2002; Menkir et al., 2007; Bersissa et al., 2011). Nonetheless, a higher prevalence of strongyles infection was recorded in the midland and highland than the lowland, and in wet season than the dry season. The mean fecal egg count was found to be significantly higher in the midland area and in wet season (Nejib et al., 2016). However, according to a study by Abebe and Esayas (2001) in the arid and semiarid zones of Eastern Ethiopia revealed that during the dry seasons of the year, a greater prevalence rates of GI helminthes were recorded in sheep and goats, $95.6 \%$ and $100 \%$ respectively. Furthermore, according to an abattoir survey by Menkir et al. (2007) at 4 abattoirs located in the semi-arid zone of Eastern Ethiopia, the mean burdens of adult nematodes were generally moderate in both sheep and goats and showed patterns of seasonal abundance that corresponded with the bi-modal annual rainfall pattern, with 
highest burdens around the middle of the rainy season. There were significant differences in the mean worm burdens and abundance of the different nematode species between the four geographic locations, with worm burdens in the Haramaya and Harar areas greater than those observed in the Dire Dawa and Jijiga locations (Abebe and Esayas, 2001; Menkir et al., 2007; Abebe et al., 2010; Berssisa et al., 2011; Endale et al., 2013). The seasonality of the GI helminthes distribution is associated with the relative humidity and rainfall (Debela, 2002; Menkir, 2007). A number of reports throughout the country indicated that there are remarkable changes in faecal egg counts and prevalence of helminths infection as a result of seasonal variation and seasonal rainfall pattern (Fikru et al., 2006; Menkir et al., 2007; Takele et al., 2013). Furthermore, relatively higher GI Helminths egg counts were found in mid altitude and highland zones than in lowland due to the influence of existing fluctuations in geographic and climatic conditions between each zone (Demelash et al., 2006; Takele et al., 2013).

\section{Epidemiological factors}

The epidemiology of the GI helminthiasis relies on factors such as the infection pressure in the environment and the susceptibility of the host, species and pathogen factor (Tilahun, 1995). From epidemiological point of view, the infective stages which eventually become available to the host depend on the independent and interactive influences of several factors in the macro- and micro-environment (Urquhart et al., 1994; Woledemariam, 2005; Regassa et al., 2006; Takele et al., 2013). Parasitic, host and environmental factors are the most frequently reported determinants for the epidemiology of helminths (Etana, 2002; Fikru et al., 2006; Ahmed et al., 2007; Menkir, 2007; Shimelis et al., 2011; Yirsaw and Zewdu, 2015). As stated by Shimelis et al. (2011), the prevalence of helminthiasis at species level was about $\mathbf{4 6 . 0 7 \%}$ and $55 \%$ in sheep and goats, respectively. Almost similar report was documented in and around Ambo town with the proportion of $\mathbf{4 7 . 8 \%}$ and $\mathbf{5 3 . 3 \%}$ in sheep and goats, correspondingly (Temesgen and Walanso, 2015). Among the collected articles, the highest post mortem examination result was reported in Eastern Ethiopia (100\%) (Abebe and Esayas, 2001). Strong association between GI helminths and poor body condition was coupled with heavy intensity of infection in the majority of infected animals (Abebe et al., 2010; Diriba et al. 2013; Alula et al., 2013; Temesgen and Walanso, 2015). According to Abebe et al. (2010) and Cheru et al. (2014), the burden of GI parasites and total EPG was significant in different body conditions.

\section{Host factor}

Sex, age, breed, nutrition, physiological status and presence or absence of inters- infections aggravate the severity of infection (Demelash et al., 2006; Menkir et al., 2007).Clinical parasitic gastroenteritis has been reported in young animals whilst infections in mature animals are generally subclinical in nature (Thomas et al., 2007). The lower occurrence in adults has been attributed to immunological maturity as the animals grow and the increase in acquired resistance due to repeated exposure (Biffa et al., 2004). While, some local breeds are known to be genetically resistant to Glhelminths infections than others (Tibbo, 2006). In Ethiopia, the local sheep breeds (Washera, Farta, Afar, Menz, Horo); Goat breeds (Begait, Abewrgelie, Keffa,) and Cattle breeds (Boran , Fogera, Raya, Horro, Abigar, Shekko, Arssi) are relatively resistant to GI worms than exotic breeds (Frisch and O'Neill, 1998; Negussie et al., 2000; Tibbo, M. 2006; Menkir et al., 2007; Solomon et al., 2009; Kebede et al., 2012). In addition, Moti et al. (2013) reported that Physiological status of ruminants like level of host immunity to the parasites is subjected to the number of eggs produced by adult female helminthes. In the same way, the females are readily infected and existing worm burdens become more active and increase egg spassed in the feces and develop Larvae ( $\left.\mathrm{L}_{3}\right)$ on the pasture (Woldemariam, 2005).

\section{Environmental factor}

As far as Ethiopia is among the tropical African countries, the temperature is permanently favorable for larval development in the environment. The favorable environment for larvae development is ranged at temperature about 10$36{ }^{\circ} \mathrm{C}$ and humidity proportion of $85 \%$ (Debela, 2002). In the arid tropical climates of lowland areas of the country has an environment which ranges from extensive pasturelands and browse plants to intensive grazing areas (Nejib et al., 2016). This environment is ranged from harsh to favorable for growth and survival of free-living stages of the GI helminthes (Tilahun, 1995; Debela, 2002).

\section{Pathogen factor}

The epidemiology of GI helminthes is also strongly influenced by host-parasite biology after infection has been occurred (Abebe et al., 2010; Diriba et al., 2013). Hypobiosis has been undergone by Gl helminthic the abomasal or intestinal mucosae of the host (Cheru et al., 2014). Whereas, Abebe and Esayas (2001) stated that the immune status of the host influenced the rates of hypobiosis and usually arrested during external environments are unfavorable for the development and survival of eggs and larvae. Such development cycle usually coincides with the onset of rainy seasons and favourable period for larval development and transmission (Kumsa and Wossenie, 2006; Feyisa et al., 2010; Hailu et al., 2011; Nejib et al., 2016).

\section{Currently applied diagnostic techniques}

The diagnosis of helminthes of ruminants is based on demonstrating the presence of eggs or larvae in fecal samples or parasites recovered from the digestive tracts or other viscera of the animals (Hailu et al., 2011; Addisu and Berihun, 2014). Although a great variety of methods and modifications have been described fordiagnosis, standardized techniques such as egg or larval counts, worm counts and pasture larval counts did not exist. Therefore, most diagnostic laboratories 
as well as teaching and research institutions were applied their own set of protocols and test procedures (Kassai, 1999). Common diagnostic procedures for helminthes infections in Africa in general and in Ethiopia in particular are simple flotation, sedimentation, modified McMaster and faecal culture methods (Hansen and Perry, 1994; Kassai, 1999; Waller, 1997). Some nematode genera such as strongyloides produce eggs that are identical in appearance which couldn't be identified easily by faecal examination alone. So to identify these faecal cultures are required (Hansen and Perry, 1994; Urquhart et al., 1996; Kassai, 1999; Van Wyket al., 2004). However, Nematodirus, Strongyloides and Trichurisspecies have eggs that can be differentiated by their distinctmorphological features. Post-mortem examinations and identification of adult worms and arrested larvae in animals are the definitive means of identifying the parasite. Similar to faecal egg counts; there are many procedures that are described for post-mortem examination for nematode parasites (Hansen and Perry, 1994; Urquhart et al., 1996; Kassai, 1999).

\section{Chemotherapies and control options}

Effective helminthic control is a major element in ensuring the sustainability of animal production. The main aim of control is therefore to ensure that the biotic potential of a parasite is restrained at a level compatible with the biological requirements of economic livestock production (Waller, 1997). Since eradication of gastrointestinal parasites is not practical, only integrated control methods can be envisaged. Some of the basic principles include grazing management, acquisition of natural or artificially induced immunity, biological control and the judicious use of anthelmintic (Hazelby et al., 1994).The main methods for control of helminthic parasites are prophylactic treatment with antihelmintics combined with grazing management (Van Wyk et al., 1999). Despite the accumulation of drugs in animal products and undesirable effects on non-target organisms in the environment, together with an increase in anthelmintic resistance, the use of antihelmintics still remains the corner-stone of helminth control (Waller, 1997; Van Wyk et al., 1999; Bersissa and Girma, 2009). Since animals are often infected with a wide range of helminths, the need for broad-spectrum compounds against trematodes, cestodes and nematodes, and their larval stages is obvious (Hazelbyet al., 1994). The epidemiological information on GI helminths parasites of domestic ruminants gathered in Ethiopia can be used to design appropriate control measures. In principle, control should aim at the reduction of transmission rates. Several control methods, which include cultural husbandry, chemical, biological, ethno-veterinary medicine and immunological control, have been proposed (Nejib et al., 2016).

\section{Husbandry pattern, control and prevention}

A thorough husbandry practices such as controlling stocking rates, rotational grazing, and providing hygienic grazing can be considered as an alternative husbandry control technique (Diriba and Birhanu, 2013; Abebe et al., 2015; Temesegen and Walanso, 2015). The best way to prevent GI helminthes is to keep animals away from potentially dangerous environment. An absolute separation of stock from intermediate host zone is only practical in intensive farming husbandry systems (Woldemariam, 2005) which the country has a limited effort to do so. However, in communal grazing condition which is very common and traditional in Ethiopia, animals are communally grazed and therefore; practices such as rotational grazing and provision of clean pastures would not be feasible (Menkir et al., 2007; Cheru et al., 2014).

\section{Chemotherapeutic interventions}

Nowadays, the control of GI parasites of livestock in Ethiopia is mainly based on the use of anthelmintics. The most commonly used generic broad spectrum antihelminthics that are available in Ethiopia are the Benzimidazoles, Imidazothiazoles and Macrocyclic lactones which consist albendazole, levamisole and ivermectin, respectively (Woldemariam, 2005; Kumsa, and Wossene, 2006; Menkir et al., 2007). It is readily available wherever in the country since it has been imported massively by the government and non-government institutions; and used by every individual including farmer. However, the use of antihelmintics at regular intervals for a long period of time and treating by mass whenever an animal manifest clinical syndrome has become the major issue for the development of multiple resistances; for instance against benzimidazoles (Woldemariam, 2005). Targeted or selective application of anthelmintic treatment might be an important tool to keep susceptible GI nematode strains in livestock and to delay this case. An alternative approach for selective anthelmintic treatment was studied using experimental small ruminants for the management of haemonchosis by using the FAMACHA ${ }^{\odot}$ method in the Mid-Rift Valley of Ethiopia by Woldemariam (2005) and Menkir (2007). Such system can be used by the farmers themselves by checking their animals for signs of anaemia (VanWyketal, 2004). Unfortunately, it was not practiced very well in Ethiopia where mixed parasitc infection, and where traditional feeding practice is followed (VanWyk et al., 2004; Kumsa and Wossene, 2006). A cost-effective preventive control programs for helminth infection in ruminants is based on sound epidemiological knowledge of the time relationship between contamination of pastures and the seasonal availability of infective larvae in a given geographic area. Epidemiological knowledge, its application in grazing management whenever feasible and access to antihelmintics of high efficacy are key factors for the success of controlling helminth infections in domestic ruminants (Aynalem et al., 2009; VanWykand Mayhew, 2013). Projecting models derived from more complete information on the ecology of GI helminth infection and anthelmintic resistance, climate and local management factors provide a basis for improved control schemes based on chemotherapy, management and immunization within similar climate (VanWyk and Mayhew, 2003; Woldemariam, 2005; Menkir et al, 2007). 


\section{Ethno-veterinary practices}

As a result of the gradually increasing anthelmintic resistance, residual effect on animal products, environmental pollution, scarcity and high cost of such drug especially to poor farmers have enforced to reconsider other alternative helminthic control technique in the country (Bersissa and Girma, 2009). Of these, Ethno-veterinary medicine has become a substantial and most expanding interest of options for Ethiopian farmers. Although such a kind of conventional veterinary medical system was yet very poor in the country, a very limited effort have been done to encourage the widely used ethno-veterinary plants in the country (Lulekal et al., 2008; Fullas, 2010; Asfaw and Fentahun, 2020). In order to do so, several Ethno-veterinary surveys were conducted so far in the country which indicated as if several traditional healers use medicinal plants for de-worming livestock (Jemal et al., 2011). To mention few examples, Herbal preparations from fresh leaves of Dodonea viscose, Albizia gummifera and Vernonia amygdalina against mixed natural infections in sheep was evaluated by Biffa et al. (2004) to show the anthelmintic activities. In addition, an In-vitro antihelmintics activity study from Rhus glutinnosa, Syzygium guineensa, Albizia gummifera, Croton macrostachyus, Ekebergia capensis, Acacia nilotica and Terminalia schimperiana against Haemonchis contortus have been reported by Eguale et al. (2006) and Jemal et al. (2011). Furthermore, anthelmintic activity of plants such as Allium sativum, Zingiber officinale, Cucurbita mexicana, Ficus religiosa, Artemisia brevifolia, Calotropis procera, Nicotiana tabacum, Butea monosperma, Coriandrum sativum, Ocimum, Thymus schimperi and Echinops kebericho have been reported by Abera (2003), Biffa et al. (2004) and Giday et al. (2007).

\section{CONCLUSION}

This review work assessed the GI helminthes in Ethiopia and provides a clue on perspectives and constraints encountered in researches which were done on GI helminths in ruminants. Twenty three (23) GI helminthes species that belong to all the three major classes of helminths have been found to occur in domestic ruminants in Ethiopia. In addition, nematodes are the most commonly encountered GI helminths while Cestodes are the least. In most reports, a higher rate was recorded in small ruminants. The most prevalent genera of GI helminths reported in order of prevalence are Haemonchus, Trichostronglyus, Oesophagostomum, Nematodirus, Cooperia, Toxocara and Bunostomum from Nematodes; Moneziaand Cyticercus from Cestodes. Whereas, Fasciola, Paramphistomum and Shistosoma are found from Trematode category. Both the abattoir and coprological studies have indicated that infection by GI helminthes in ruminants is highly prevalent and widespread in all agro-ecologies and livestock production systems in Ethiopia. Fecal diagnostic techniques such as simple flotation, sedimentation, modified McMaster have been used routinely in Ethiopia. It has been also shown that prevalence of GI helminthes parasites was related to the agro-climatic conditions such as quantity and quality of pasture, temperature, humidity and grazing behavior of the host and the susceptibility of any intestinal helminthic parasites were also influenced by age, breed, species, health status, physiological factors and previous exposure to parasites.

Due to the lack of effective helminthes control strategies in Ethiopia, antihelmintics are exclusively used. Though Ethiopia has a huge amount of small and large ruminants population, the country is facing a direct and indirect economic lose as a result of GI helminthes infection. Hence, immediate remedies shall be taken into action on control and prevention methods against such anthelmintic resistant GI parasites. In order to this, it is advantageous to collect and looking over the previous researches done so far to reconsider their gaps for the future short and long term actions on prevention and control strategies. So, all-inclusive and well organized documentation about GI helminthes of ruminants in the country is essential to support researchers and policy makers to develop such remedies. Finally, applicable field diagnostic technique should be introduced as far as mixed parasitic infection and traditional feeding practice is common in the country which can aggravate GI helminthes infection.

\section{DECLARATIONS}

\section{Consent to publish}

Not applicable.

\section{Competing interests}

The authors declare that they have no competing interests.

Funding

This study was funded by the University of Gondar.

Avallability of data and materials

Data will be made available up on request of the primary author

\section{Acknowledgment}

The authors' heartfelt thanks the University of Gondar, Research and Community Service V/President for the financially supporting in the study. 


\section{REFERENCES}

Abebe R, Mebrahtu G, Solomon M, Fufa A, and Alemayehu R (2010). Gastrointestinal nematode infections in small ruminants under the traditional husbandry system during the dry season in southern Ethiopia. Tropical Animal Health and Production, 42:1111-1117. Doi: https://doi.org/10.1007/s11250-010-9532-3

Abebe TG, Belay M, Shahid N and Assefa A (2015). Major metacestodes in small ruminants slaughtered at Dessie municipal abattoir, Eastern Ethiopia: Prevalence, cyst viability, organ distribution and economic implications. Comparative Clinical Pathology, 24: 659668. Doi: https://doi.org/ 10.1007/s00580-014-1964-0.

Abebe W, and Esayas G (2001). Survey of Ovine and Caprine Gastro-intestinal helminthosis in eastern part of Ethiopia during the dry season of the year. Revue de Médecine Vétérinaire, 152 (5), 379-384. Link: https://www.revmedvet.com/2001/RMV152_379_384.pdf

Abera B (2003). Medicinal plants used in traditional medicine in Jimma Zone, Southwest Ethiopia. Ethiopian Journal of Health Sciences, 13: 85-94. Link: https://www.ajol.info/index.php/ejhs/article/view/146241

Addisu B, and Berihu H (2014). Study on Prevalence of Gastrointestinal Nematodes and Coccidian Parasites Affecting Cattle in West Arsi zone, Ormia Regional State, Ethiopia. Journal of Veterinary Science and Technology, 5(5): 1000207. Link

Ahmed EF, Markvichitr K, Tumwasorn S, Koonawootrittriron S, Choothesa A, Jittapalapong S (2007). Prevalence of Fasciola spp. Infection of sheep in the Middle Awash River Basin, Ethiopia. Southeast Asian Journal of Tropical Medicine and Public Health, Vol 38 (suppl 1): 51 57. Link: http://citeseerx.ist.psu.edu/viewdoc/download?doi=10.1.1.1067.4234\&rep=rep1\&type=pdf

Alula P, Addisu K, and Amanuel W (2013). Prevalence and economic significance of Bovine Fasciolosis in Nekemte Municipal abattoir. Journal of Veterinary Medicine and Animal health, 5(8): 202-205. https://academicjournals.org/article/article1379686620_Petros\%20et\%20al.pdf

Asfaw M and Fentahun T (2020). Treatment trials of epizootic lymphangitis with local medicinal plants: a review. Online Journal of Animal and Feed Research, 10(4): 93-101. Link

Ayalew S, and Endalkachew N (2013). Prevalence and Risk Factors of Bovine and Ovine Fasciolosis, and evaluation of direct sedimentation sensitivity method at Bahir Dar Municipal Abattoir, Northern Ethiopia. Ethiopian Veterinary Journal, 17(2):1-17. Doi: https://doi.org/10.4314/evj.v17i2.1

Aynalem H, Abebaw G, Taye T, and Markos T (2009). Epidemiology of nematode parasites of sheep around Jimma, southwestern Ethiopia. Tropical Animal Health and Production, 42: 825-831. Doi: https://doi.org/10.1007/s11250-009-9492-7

Bekele T, Mukasa-Mugerwa E and Kasali OB (1988). The prevalence of cysticercoids and hydatidosis in Ethiopian sheep. Veterinary Parasitology, 28: 267-270. Doi: https://doi.org/10.1016/0304-4017(88)90114-8

Bekele T, Woldeab T, Lahlou-Kassi A, and Sherngton J (1992) .Factors affecting morbidity and mortality on farm and on station in Ethiopia highland sheep. Acta Tropica, 52: 99-109. Doi: https://doi.org/10.1016/0001-706X(92)90025-S

Bersissa K and Girma A (2009). Multiple anthelmintic resistance on a goat farm in Hawassa (southern Ethiopia). Tropical Animal Health and Production , 41: 655-662. Doi: https://doi.org/10.1007/s11250-008-9237-z

Bersissa K, Tigist T, Teshale S, Reta D, and Bederu H (2011). Helminthes sheep and goats in central Oromia (Ethiopia).During the dry season. Journal of Animal and Veterinary Advances, 10(14): 1845-1849. Link

Beyene T and Hiko A (2019). Zoonotic metacestodes and associated financial loss from cattle slaughtered at Yabello municipal abattoir, Borana-Oromia, Ethiopia. Parasite Epidemiology and Control, 5: e00096. Doi: https://doi.org/10.1016/j.parepi.2019.e00096

Biffa D, Nurfeta A, and Jobre Y (2004). Evaluation of anthelmintic activities of crude leaf extracts of three indigenous herbal plants against ovine gastrointestinal nematodes. Ethiopian Veterinary Journal, 8: 57-58.

Cheru T, Birhanu A, Diriba L, and Eyob E (2014). Prevalence of gastrointestinal parasitism of cattle in East Showa Zone, Oromia Regional State, Central Ethiopia. Journal of Veterinary Medicine and Animal Health, 6(2): 54-62. Link: https://academicjournals.org/journal/JVMAH/article-full-text-pdf/DODC71C42793

CSA (2013). Agricultural Sample Survey, 2012/13, Volume II: Report on Livestock and livestock characteristics (Private peasant holdings). Statistical Bulletin 570. Central Statistical Agency (CSA), Federal Democratic Republic of Ethiopia, Addis Ababa.

Debela E (2002). Epidemiology of gastrointestinal helminthiasis of Riff valley goats under traditional husbandry system in Adami Tulu district. Ethiopia. Ethiopian Journal of Sciences. 25, 35-44. Link: https://www.ajol.info/index.php/sinet/article/view/18072

Demelash B, Yilma J, and Hassen C (2006). Ovine helminthosis, a major health constraint to productivity of sheep in Ethiopia. Animal Health Research Reviews, 7(1/2),107-118. Doi: https://doi.org/10.1017/s1466252307001132

Diriba L, and Birhanu A (2013). Prevalence of ovine gastrointestinal nematodes in and around Asella, South Eastern Ethiopia. Journal of Veterinary Medicine and Animal Health, 5(8): 222-228. Link

Eguale T, Tilahun G, Gidey M, and Mekonnen Y (2006). In-Vitro anthelmintic activities of four ethiopian medicinal plants against Haemonchus contortus. Pharmacologyonline, 3:153-165. Link I Google Scholar

Elsa L, Sofia V, Elvira S, Maria M, Mendes G, and André M (2012). Factors Influencing Livestock Productivity. Environmental Stress and Amelioration in Livestock Production, Springer-Verlag Berlin Heidelberg. Link

Endale M, Shihun S, Jemere B, and Desie S (2013). Sheep and goats Cysticercus tenuicollis prevalence and associated risk factors. African Journal of Agricultural Research, 8(24): 3121-3125. doi: https://doi.org/10.5897/AJAR2012.7361

Etana D (2002). Epidemiology of Gastrointestinal helminthes of Rift valley goats under traditional husbandry systems in Adami Tulu District, Ethiopia. Ethiopian Journal of Science, 25(1): 35-44. Link: https://www.ajol.info/index.php/sinet/article/view/18072

Feyesa R, Alemante M, and Jemere B (2010). Study on the prevalence of cystic hydatidosisand its economic significance in cattle slaughteredat Hawassa Municipal abattoir, Ethiopia. Tropical Animal Health and Production, 42: 977-984. Doi: https://doi.org/10.1007/s11250-009-9517-2

Fikru R, Yosef K, Teshale S, Reta D, and Yosef K (2006). Epidemiology of Gastrointestinal Parasites of Ruminants in Western Oromia, Ethiopia. International Journal Applied Reesearch and Veterinary Medicine, 4:51-57. Link

Frisch JE, and O'Neill CJ (1998). Comparative evaluation of beef cattle breeds of African, European and Indian origins. 1. Live weights and heterosis at birth, weaning and 18 months. Animal Science, 67: 27-38. Doi: https://doi.org/10.1017/S1357729800009760

Fufa A, Sisay F, Bekele M, and Alemayehu R (2012). Prevalence of bovine hydatidosis in Kombolcha ELFORA abattoir, North Eastern Ethiopia. Open Journal of Animal Sciences, 4:281-286. http://dx.doi.org/10.4236/ojas.2012.24038.

Fullas F (2010). Ethiopian medicinal plants in veterinary healthcare.A mini-review. Ethiopian eJournal for Research and Innovation Foresight, 2: 48-58. Link 
Getachew A and Ashwani K (2013). Cysticercosis in cattle and its public health implications in Mekelle City and surrounding areas, Ethiopia. Ethiopian Veterinary Journal, 17: 31-40. Link: https://www.ajol.info/index.php/evj/article/download/99352/88643

Getawa A, Beyene D. Ayana D, Megersa B, and Abunna F (2010). Hydatidosis: Prevalence and its economic importance in ruminants slaughtered at Adama municipal abattoir, Central Oromia, Ethiopia. Acta Tropica, 221-225. Doi: https://doi.org/10.1016/i.actatropica.2009.10.019

Giday M, Teklehaymanot T, Animut A, and Mekonnen Y (2007). Medicinal plants of the Shinasha, Agew Awi and Amhara peoples in northwest Ethiopia. Journal of Ethnopharmacology, 110: 516-525. Doi: https://doi.org/10.1016/j.jep.2006.10.011

Hailu D, Cherenet A, Moti Y, and Tadele T (2011).Gastrointestinal Helminth Infections in Small-Scale Dairy Cattle Farms of Jimma Town, Ethiopia. Ethiopean Journal of Applied Sciences and Technology 2(1): 821. Link

Hansen J, and Perry B (1994). The epidemiology diagnosis and control of helminth parasites of ruminants.A Handbook. International Laboratory for Research on Animal Diseases, Nairobi, Kenya. Link: https://cgspace.cgiar.org/handle/10568/49809

Hazelby, CA, Probert, AJ, and Rowlands DAP (1994). Anthelmintic resistance in nematodes causing parasitic gastroenteritis of sheep in the UK. Journal of Veterinary Pharmacology and Therapeutics. 17(4): 245-252. Doi: https://doi.org/10.1111/j.13652885.1994.tb00240.x

Jemal H, Kaba U, Fayissa R, Awol J, Sultan A, and Nezif H (2011). Antihelmintic Effect of the Essential Oil Extracts of Selected Medicinal Plants against Haemonchus contortus. International Journal of Agricultural Research, 6: 290-298. Doi: http://dx.doi.org/10.3923/ijar.2011.290.298

Jibat T, Ejeta G, Asfaw Y, and Wudie A (2008). Causes of abattoir condemnation in apparently healthy slaughtered sheep and goats at HELMEX abattoir, Debre Zeit, Ethiopia. Revue de Médecine Vétérinaire, 159(5): 305-311. Link: https://www.revmedvet.com/2008/RMV159 305 311.pdf

Kassai T (1999). Veterinary Helminthology. Reed Educational and professional publishers, Oxford UK, 260.

Kebede T, Haile A, Dadi H (2012). Smallholder goat breeding and flock management practices in the central rift valley of Ethiopia. Tropical Animal Health and Production, 44: 999-1006. Doi: https://doi.org/10.1007/s11250-011-0033-9

Kebede W, Hagos A, Girma Z, Lobago F (2009). Echinococcosis/hydatidosis: its prevalence, economic and public health significance in Tigray region, North Ethiopia. Tropical Animal Health and Production, 41: 865-871. Doi: https://doi.org/10.1007/s11250-008-9264$\underline{9}$

Khan MK, Sajid MS, Khan MN, Iqbal Z, Iqbal MU (2009). Bovine Fasciolosis: Prevalence, effects of treatment on productivity and cost benefit analysis in five districts of Punjab, Pakistan. Research in Veterinary Science, 87: 70-75. Doi: https://doi.org/10.1016/i.rvsc.2008.12.013

Kumsa B, and Wossene A (2006). Abomasal nematodes of small ruminants of Ogaden region, eastern Ethiopia: Prevalence,worm burden and species composition. Revue de Médecine Vétérinaire, 157(12): 27-32. Link

Lebbie SH, Rey B, and Irungu EK (1994). Small ruminant research and development in Africa Proceedings of the second biennial conference of the African small ruminant research network. ILCA, pp.1-5. Link

Lemma B, Gebreab F, and Tedla S (1985). Studies on fascioliasis in four selected sites in Ethiopia. Veterinary Parasitology, 18: 29-37. DOI: https://doi.org/10.1016/0304-4017(85)90005-6

Lidya G, and Berihun AT (2015). Prevalence of Ovine Haemonchosis in Wukro, Ethiopia . Hindawi Publishing Corporation Journal of Parasitology Research, Article ID: 635703. Doi: http://dx.doi.org/10.1155/2015/635703.

Lulekal E, Kelbessa E, Bekele T, and Yineger H (2008). An ethno-botanical study of medicinal plants in Mana Angetu District, southeastern Ethiopia. Journal of Ethnobiology and Ethnomedicine, 4:10. Doi: https://doi.org/10.1186/1746-4269-4-10

Menkir MS, Arvid U, and Peter Waller J (2007). Prevalence and seasonal incidence of nematode parasites and fluke infections of sheep and goats in eastern Ethiopia. Tropical Animal Health and Production, 39: 521-531. Doi: https://doi.org/10.1007/s11250-007-9035-z

Menkir S (2007). Helminth Parasites of Sheep and Goats in Eastern Ethiopia: Epidemiology, and Anthelmintic Resistance and its Management. Doctoral thesis, Faculty of Veterinary Medicine and Animal Science, Swedish University of Agricultural Sciences, Uppsala, Sweden. Link: https://core.ac.uk/download/pdf/11695158.pdf

Moti Y, Derara B, Dalasa D, Senbeta T, and Hailu D (2013). Bovine Trypanosomosis and gastrointestinal helminthosis in settlement villages of Bedele district, South- western Ethiopia. Ethiopian Veterinary Journal, 17(1):41-54. Doi: https://doi.org/10.4314/evj.v17i1.4

Negussie E, Rottman OJ, Pirchner F, Rege JE (2000). Allometric growth coefficients and partitioning of fat depots in indigenous Ethiopian Menz and Horro sheep breeds. In The Opportunities and Challenges of Enhancing Goat Production in East Africa. Workshop Proceedings. Langston University, OK (USA). E (Kika) dela Garza Inst. for Goat Research. Langston, OK (USA) 2000 (pp. 151-163). Google Scholar

Nejib M, Messele T, Amenu A, and Desie S (2016). Epizootological study of small ruminant gastrointestinal strongyles in Gamo-Gofa Zone, Southern Ethiopia. Journal of Parasitic Disease, 40(2): 469-474. DOI: https://dx.doi.org/10.1007\%2Fs12639-014-0528-1

Nigatu K, Abebe M, and Getachew T (2009). Hydatidosis of slaughtered animals in Bahir Dar Abattoir, Northwestern Ethiopia. Tropical Animal Health and Production, 41:43-50. Doi: https://doi.org/10.1007/s11250-008-9152-3

Njau BC, Scholtens RG and Kasali $O$ (1990). Parasites of sheep at the International Livestock Centre for Africa Debre Berhan Station, Ethiopia. Preventive Veterinary Medicine, 9: 267-277. Doi: https://doi.org/10.1016/0167-5877(90)90072-P

Regassa A, Abunna F, Mulugeta A, and Megersa B (2009). Major metacestodes in cattle slaughtered at Wolaita Soddo Municipal abattoir, Southern Ethiopia: Prevalence, cystviability, organ distribution and socioeconomic implications. Tropical Animal Health and Production, 41: 1495-1502. Doi: https://doi.org/10.1007/s11250-009-9338-3

Regassa F, Sori T, Dhuguma R, and Kiros $Y(2006)$. Epidemiology of gastrointestinal parasites of ruminants in western Oroma, ethiope. International Journal Applied Research of Veterinary Medicine, 4(1): 51-57. Doi: https://www.jarvm.com/articles/Vol4Iss1/Vol4Iss1RegassaV4N1pp51-57.pdf

Shimelis D, Asmare A, and Wudu T (2011). Epidemiology of gastrointestinal helminthiasis of small ruminants in selected sites of North Gondar zone, Northwest Ethiopia. Ethiopian Vetrinary Journal 15 (2): 57-68. Doi: https://doi.org/10.4314/evj.v15i2.67694

Solomon G, Komen V, Arendonk JAM (2009). Optimal village breeding schemes under smallholder sheep farming systems. Livestock Science, 124: 82-88. Doi: https://doi.org/10.1016/j.livsci.2009.01.001

Takele S, Yacob H, and Getachew T (2013). Epidemiology of gastrointestinal nematodes of Horro sheep in Western Oromiya, Ethiopia. Journal of Veterinary Medicine and Animal Health, 5(10): 296-304. Doi: https://doi.org/10.5897/JVMAH2013.0234 
Tembely S, and Hansen JW (1996). Helminth Diseases of Small Ruminants in the Tropics: A Review of Epidemiology and Control Strategies, Bogor, Indonesia, 1996: 123-127. Link

Temesgen A, and Walanso I (2015). Major gastrointestinal helminth parasites of grazing small ruminants in and around Ambo town of Central Oromia, Ethiopia. Journal of Veterinary Medicine and Animal Health, 7: 64-70 . Doi: https://doi.org/10.5897/JVMAH2014.\%200327

Tesfaye H (1998). Ovine and bovine helminthiasis in Kelala (South Wollo). Ethiopian Vet Association Proceedings of the 12 ${ }^{\text {th }}$ Conference. Addis Ababa, Ethiopia, pp. 30-34.

Thomas N, Teshale S, and Kumsa B (2007). Abomasal nematodes of sheep and goatsslaughtered in Awassa (Ethiopia): Species composition, prevalence and vulvar morphology. Helminthologia, 44: 70-75. Doi: https://doi.org/10.2478/s11687-007-0006-8

Tibbo M (2006). Productivity and health of indigenous sheep breeds and crossbreds in the central Ethiopian highlands. Doctoral dissertation. ISSN 1652-6880, ISBN 91-576-7100-1. Link: https://core.ac.uk/download/pdf/11695029.pdf

Tilahun G (1995). Epidemiology of helminth parasites of small ruminants in midlowland Ethiopia. In: Gray GD, Uilenberg G, Jacobsen LL (Eds.), Parasitology Research in Africa. Proceedings of an IFS (International Foundation for Science) workshop, Bobo Dioulasso, Burkina Faso. pp. 255-269. Google Scholar

Tilahun H, and Schmidt E (2012). Spatial analysis of livestock production patterns in Ethiopia. ESSP II Working Paper 44. International Food Policy Research Institute/Ethiopia Strategy Support Program II, Addis Ababa, Ethiopia. Link: https://www.ifpri.org/publication/spatialanalysis-livestock-production-patterns-ethiopia

Urquhart GM, Armour J, Duncan JL, Dunn AM, Jennings FW (1996). Veterniary Parasitology 2nd Edn, Oxford, Longman Scientific and Technical Press, UK. 100-109.

Van Wyk JA, and Mayhew E (2013). Morphological identification of parasitic nematode infective larvae of small ruminants and cattle: A practical lab guide. Onderstepoort Journal of Veterinary Research, 80: E1-E14. Link

Van Wyk JA, Cabaret J, and Michael LM (2004). Morphological identification of nematodes of small ruminants and cattle simplified. Veterinary Parasitology, 119: 277-306. Doi: https://doi.org/10.1016/j.vetpar.2003.11.012

Van Wyk JA, Malan FS and Randles JL (1999). How long before resistance makes it impossible to control some field strains of Haemonchuscontortus in South Africa with any of the modern anthelmintics. Veterinary Parasitology, 70: 111-122. Doi: https://doi.org/10.1016/S0304-4017(96)01147-8

Waller PJ (1997). Sustainable helminth control of ruminants in developing countries. Veterinary Parasitology, 71: 195-207. Doi: https://doi.org/10.1016/s0304-4017(97)00032-0

Winter J, Rehbein S, and Joachim A (2018). Transmission of helminthes between species of ruminants in Austria appears more likely to occur than generally assumed. Frontiers in Veterinary science, 5: 30. Doi:https://doi.org/10.3389/fvets.2018.00030

Woldemariam DL (2005). Nematode prevalence, helminths management practices and anthelmintic resistance in small ruminants in the mid-rift valley of Ethiopia. Thesis, Faculty of Veterinary Science, and University of Pretoria, South Africa. Google Scholar

Yirsaw K, and Zewdu S (2015). Bovine and ovine schistosomiasis: prevalence and associated risk factors in South Achefer. The 25th Staff and students annual research conference, June 19-20, 2015. Gondar, Ethiopia. Google Scholar

Yitayal G, Mebrehatu G, and Abebe T (2015). Prevalence of Bovine Fasciolosis in and around Bahir Dar, Northwest Ethiopia. Journal of Parasitology and Vector Biology, 7(4): 74-79. Link: https://academicjournals.org/article/article1429098988_Gebrie.pdf

Zahid IA, Latif M, and Baloch KB (2005). Incidence of endoparasites in exotic cattle calves. Pakistan Veterinary Journal, 25 (1): $47-48$. Link 